EPJ Web of Conferences 81, 01019 (2014)

DOI: $10.1051 /$ epjconf/ 20148101019

(C) Owned by the authors, published by EDP Sciences, 2014

\title{
First $\pi K$ atom lifetime measurement and recent results from the DIRAC experiment
}

\author{
Mikhail Zhabitsky ${ }^{1, a}$ for the DIRAC Collaboration \\ ${ }^{1}$ Joint Institute for Nuclear Research, Joliot-Curie 6, 141980 Dubna, Russia
}

\begin{abstract}
We report evidence for $\pi K$ atoms production, using $24 \mathrm{GeV} / c$ proton beam from CERN PS interacting with a thin Ni target. We have identified (178 \pm 49$) \pi K$ pairs, which were produced in a bound state $-\pi K$ atom, which was subsequently brokenup (ionized) in the Ni target. Our analysis yields a first measurement of the $\pi K$ atom lifetime $\left(2.5_{-1.8}^{+3.0}\right)$ fs [1]. This lifetime is connected in a model-independent way to the S-wave isospin-odd $\pi K$ scattering length $\left|a_{0}^{-}\right|=\frac{1}{3}\left|a_{1 / 2}-a_{3 / 2}\right|=\left(0.11_{-0.04}^{+0.09}\right) M_{\pi}^{-1}$ ( $a_{I}$ for isospin $I$ ).
\end{abstract}

\section{Introduction}

Low-energy QCD and specifically Chiral Perturbation Theory (ChPT) [2] predict $\pi \pi$ and $\pi K$ scattering lengths with high precision [3,4]. For processes involving $u$-and $d$-quarks theoretical predictions have been experimentally checked by $\pi^{+} \pi^{-}$atom lifetime measurement [5] and by analysis of $K$ decays $[6,7]$. Detection and lifetime measurement of $\pi K$ atom cast a look into processes which involve $s$-quark as well.

$A_{\pi K}(\pi K$ atom) is an exotic atom - a hydrogen-like bound system of oppositely charged $\pi$ and $K$ mesons. Its ground state binding energy is $2.9 \mathrm{keV}$, Bohr radius $a_{B}=249 \mathrm{fm}$ and Bohr momentum is $0.79 \mathrm{MeV} / c$. Lifetime of $\pi K$ atoms is limited by strong interaction through the decay into a pair of neutral pion and kaon, $\pi^{0} K^{0}$ or $\pi^{0} \overline{K^{0}}$, other decay channels contribution is of the order $10^{-3}$. The $A_{\pi K}$ decay width $\Gamma_{\pi K}$ in the ground state (1S) is directly related to the S-wave isospin-odd $\pi K$ scattering length $a_{0}^{-}=\frac{1}{3}\left(a_{1 / 2}-a_{3 / 2}\right)[8,9]$ :

$$
\Gamma_{\pi K}=\frac{1}{\tau} \simeq \Gamma\left(A_{\pi K} \rightarrow \pi^{0} K^{0} \text { or } \pi^{0} \overline{K^{0}}\right)=8 \alpha^{3} \mu^{2} p^{*}\left(a_{0}^{-}\right)^{2}\left(1+\delta_{K}\right) .
$$

Here $\tau$ is the lifetime in the ground state, $a_{I}$ is the scattering length for isospin $I, \alpha$ is the fine structure constant, $\mu$ is the reduced mass of $\pi^{ \pm} K^{\mp}$ pair, $p^{*}$ is the outgoing $\pi^{0}$ momentum in the atomic rest frame, and $\delta_{K}$ summarizes corrections due to isospin breaking.

Inserting in (1) the value $M_{\pi} a_{0}^{-}=0.090 \pm 0.005$ calculated with the dispersion analysis [10] and using $\delta_{K}=0.040 \pm 0.022$ [9] predicts the $\pi K$ atom lifetime

$$
\tau=(3.5 \pm 0.4) \cdot 10^{-15} \mathrm{~s} .
$$

DIRAC experiment aims to measure the $\pi K$ atom lifetime, thus checking the ChPT prediction.

\footnotetext{
a e-mail: Mikhail.Zhabitsky@jinr.ru
} 


\section{Experimental method}

The method of $A_{\pi K}$ lifetime measurement [11] used by DIRAC experiment is as follows. $A_{\pi K}$ originate from collisions of $24 \mathrm{GeV} / c$ protons with $\mathrm{Ni}$ atoms. Atoms are produced in $\mathrm{nS}$-states, depending on the principal quantum number as $n^{-3}$ [11]:

$$
\frac{d \sigma_{A}}{d \vec{p}_{A}}=\left.(2 \pi)^{3} \frac{E_{A}}{M_{A}} \frac{d^{2} \sigma_{s}^{0}}{d \vec{p}_{K} d \vec{p}_{\pi}}\right|_{\frac{\vec{p}_{K}}{M_{K}} \approx \frac{\vec{p} \pi}{M_{\pi}}} \cdot\left|\psi_{n}(0)\right|^{2}=\left.(2 \pi)^{3} \frac{E_{A}}{M_{A}} \frac{1}{\pi a_{B}^{3} n^{3}} \frac{d^{2} \sigma_{s}^{0}}{d \vec{p}_{K} d \vec{p}_{\pi}}\right|_{\frac{\vec{p}_{K}}{M_{K}} \approx \frac{\vec{p} \pi}{M_{\pi}}},
$$

where $\vec{p}_{A}, E_{A}$ and $M_{A}$ are the momentum, total energy and mass of the $\pi K$ atom in the laboratory system, respectively, and $\vec{p}_{K}$ and $\vec{p}_{\pi}$ the momenta of the charged kaon and pion with equal velocities. The inclusive production cross section from short-lived sources without final state interaction is denoted as $\sigma_{s}^{0}, \psi(0)$ stands for the atomic wave function at the origin.

On a similar way, free $\pi^{ \pm} K^{\mp}$ pair production from short-lived sources is enhanced by a GamowSommerfeld-Sakharov factor [12] as a function of their relative momentum $q$ :

$$
\frac{d^{2} \sigma_{C}}{d \vec{p}_{K} d \vec{p}_{\pi}}=\frac{d^{2} \sigma_{s}^{0}}{d \vec{p}_{K} d \vec{p}_{\pi}} \frac{4 \pi \mu \alpha / q}{1-\exp (-4 \pi \mu \alpha / q)}
$$

Thus the total number $N_{A}$ of produced atoms is proportional to the number $N_{C}$ of Coulomb pairs generated with small relative momentum:

$$
N_{A}=k\left(q_{0}\right) N_{C}\left(q \leqslant q_{0}\right) .
$$

While crossing the target, $\pi K$ atoms can be excited or even ionized (break-up) due to Coulomb interaction with target atoms. Pairs of $\pi^{ \pm} K^{\mp}$ mesons from $\pi K$ atom break-up, called atomic pairs later in the text, are characterized by low relative momenta $q$ in their center of mass system. Thanks to their distinct shape, atomic pairs can be revealed in a reconstructed $Q$ distribution $(Q$ stands for experimental relative momentum) by an experiment with sufficiently high momentum resolution. Thus a number $n_{A}$ of atomic pairs can be identified from the experimental $Q$-spectra over background of free $\pi K$ pairs. Ionization of $\pi K$ atoms in the target is in competition with their annihilation. Therefore the probability $P_{\mathrm{br}}=n_{A} / N_{A}$ of $A_{\pi K}$ ionization in the target is a unique function of its lifetime $\tau$, which is calculated with sufficient precision $[13,14]$ (Fig. 1).

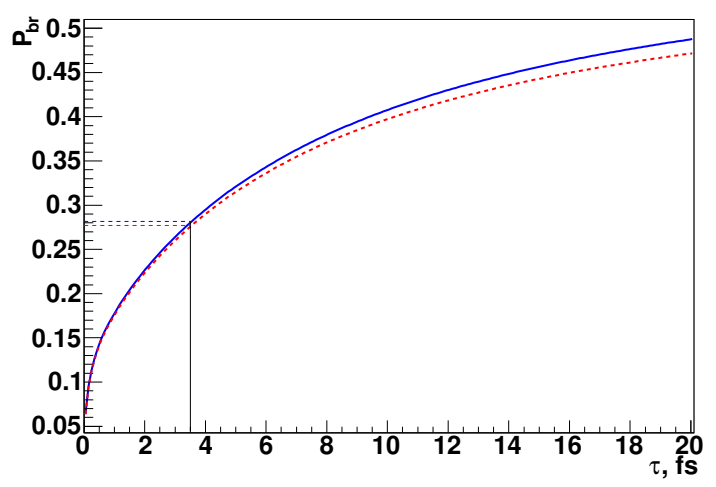

Figure 1. Probability of $A_{\pi K}$ ionization as a function of the ground state lifetime $\tau$ calculated for Ni targets of thickness $98 \mu \mathrm{m}$ (dashed red line) and $108 \mu \mathrm{m}$ (solid blue line). The theoretically predicted lifetime $\tau$ (Eq. 2) corresponds to $P_{\mathrm{br}}=0.28$.

Earlier this method was successfully applied to measure the lifetime of exotic $\pi^{+} \pi^{-}$atoms [5]. First evidence for $\pi K$ atoms through the observation of $(173 \pm 54) \pi K$ atomic pairs produced on $26 \mu \mathrm{m}$ Pt target in 2007 has been published [15]. In the current work data from Ni targets is analysed, as for Ni a function $P_{\mathrm{br}}(\tau)$ provides better sensitivity to the lifetime $\tau$. 


\section{Experimental setup}

The DIRAC Collaboration constructed the double arm magnetic spectrometer which is optimized to detect and identify $\pi^{+} \pi^{-}, \pi^{+} K^{-}$and $\pi^{-} K^{+}$pairs with small relative momenta $Q$. The sketch of the apparatus is shown on Fig. 2.

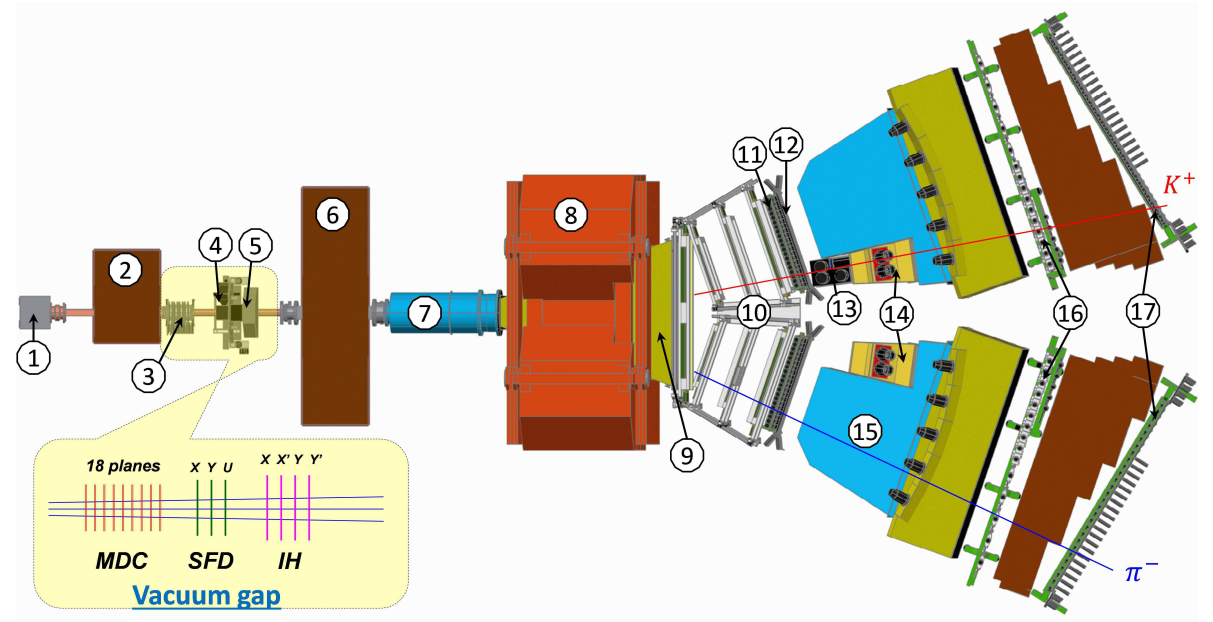

Figure 2. General view of the DIRAC setup: 1 - target station; 2 - first shielding; 3 - microdrift chambers; 4 - scintillating fiber detector; 5 - ionisation hodoscope; 6 - second shielding; 7 - vacuum channel; 8 spectrometer magnet; 9 - vacuum chamber; 10 - drift chambers; 11 - vertical hodoscope; 12 - horizontal hodoscope; 13 - aerogel Cherenkov; 14 - heavy gas Cherenkov; 15 - nitrogen Cherenkov; 16 - preshower; 17 - muon detector.

The experiment uses a $24 \mathrm{GeV} / c$ primary proton beam from the CERN PS. Protons are directed onto a pure Ni thin foil with a radiation thickness of $\sim 7 \cdot 10^{-3} X_{0}$. The DIRAC apparatus with its secondary channel is inclined by $5.7^{\circ}$ with respect to the primary proton beam. Tracking detectors are positioned in two groups: between the target station and the spectrometer magnet $(B L=2.2 \mathrm{~T} \cdot \mathrm{m})$, and downstream the magnet. The resolution of the setup on components of pair relative momenta in c.m.s. $\sigma_{Q_{x}} \approx \sigma_{Q_{y}} \approx 0.2 \mathrm{MeV} / c$ and $\sigma_{Q_{L}} \approx 0.85 \mathrm{MeV} / c$ is comparable to $A_{\pi K}$ Bohr momentum. Particle identification $(e / \mu / \pi / K / p)$ is performed through a combination of time-of-flight technique, threshold Cherenkov detectors and muon veto.

\section{Experimental results}

The data sample was collected on two Ni targets with thickness $(98 \pm 1) \mu \mathrm{m}$ in 2008 and $(108 \pm$ 1) $\mu \mathrm{m}$ in 2009 and 2010. Events containing $\pi^{ \pm} K^{\mp}$ pairs with low relative momenta $Q$ in their centreof-mass system were selected. Then the reconstructed 2-dimensional $\left(Q_{T}, Q_{L}\right)$ distribution of $\pi K$ pairs was fitted by a sum of simulated distributions of atomic, Coulomb and non-Coulomb pairs with abundances of each component as free fit parameters. Here $Q_{L}$ corresponds to a component of the relative momentum parallel to the direction of pair total momentum, while $Q_{T}$ comprises the transverse component. In the low $Q_{L}$ region (Fig. 3), where atomic pairs are expected, there is an excess of events over the background of free pairs. Fit results for all data periods are summarized in 

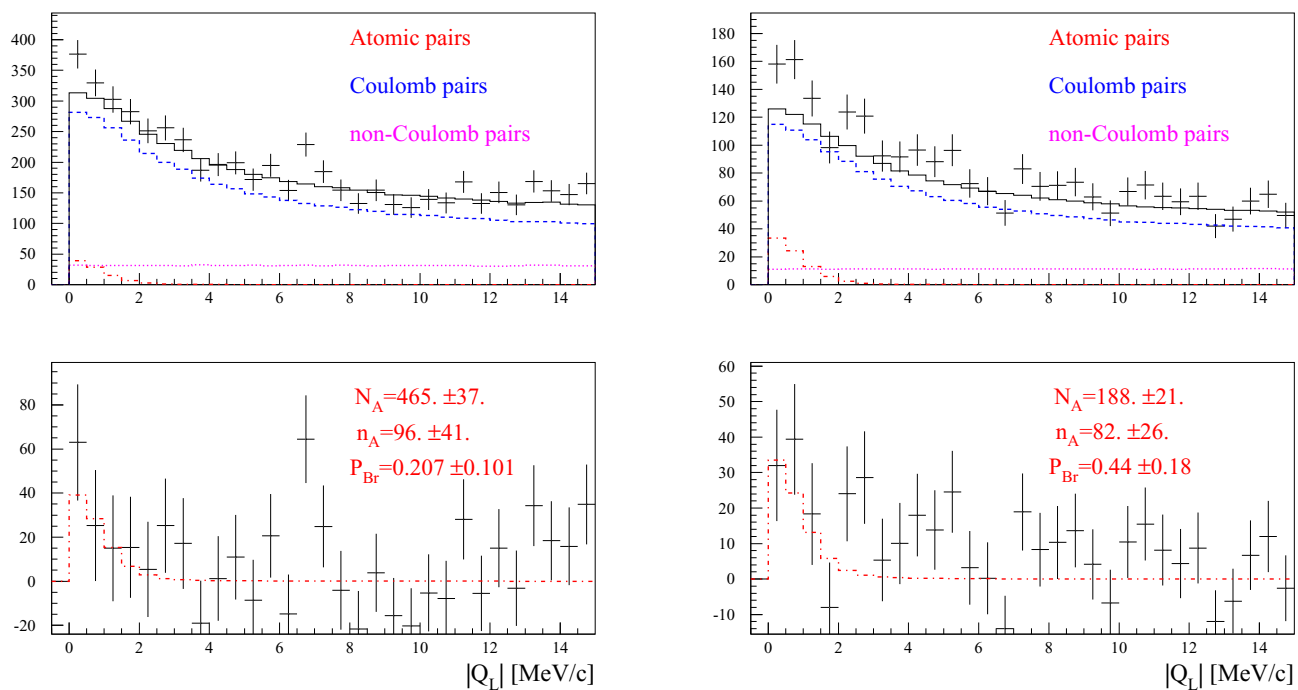

Figure 3. Experimental distributions on $\left|Q_{L}\right|$ for $\pi^{-} K^{+}$(left) and $\pi^{+} K^{-}$pairs (right). Top: experimental $\left|Q_{L}\right|$ distribution (crosses) is fitted by the sum of simulated distributions of atomic (red), Coulomb (blue) and non-Coulomb pairs (pink). The sum of free pair (Coulomb and non-Coulomb) distributions is shown by solid black line. Bottom: the difference between experimental and free pair distributions is compared to simulated distribution for atomic pairs.

Table 1. Experimental results for number of produced atoms $N_{A}$, number of observed atomic pairs $n_{A}$ and probability of break-up $P_{\mathrm{br}}$ reconstructed by $\left(Q_{T}, Q_{L}\right)$-analysis from collected data.

\begin{tabular}{rrcc}
\hline Year & \multicolumn{1}{c}{$N_{A}$} & $n_{A}$ & $P_{b r}$ \\
\hline \multicolumn{4}{c}{$\pi^{-} K^{+}$from $\left(Q_{T}, Q_{L}\right)$-analysis } \\
\hline 2008 & $132 \pm 16$ & $14 \pm 19$ & $0.11 \pm 0.15$ \\
2009 & $169 \pm 24$ & $33 \pm 26$ & $0.20 \pm 0.17$ \\
2010 & $164 \pm 23$ & $49 \pm 26$ & $0.30 \pm 0.19$ \\
All & $465 \pm 37$ & $96 \pm 41$ \\
\hline \multicolumn{4}{c}{$\pi^{+} K^{-}$from $\left(Q_{T}, Q_{L}\right)$-analysis } \\
\hline 2008 & $51 \pm 11$ & $21 \pm 13$ & $0.41 \pm 0.33$ \\
2009 & $78 \pm 13$ & $26 \pm 16$ & $0.34 \pm 0.24$ \\
2010 & $60 \pm 12$ & $35 \pm 16$ & $0.58 \pm 0.36$ \\
All & $188 \pm 21$ & $82 \pm 26$ & \\
\hline
\end{tabular}

Table 1. In total, the number of reconstructed atomic pairs of both charge combinations amounts to $n_{A}\left(\pi^{-} K^{+}+\pi^{+} K^{-}\right)=178 \pm 49$.

The lifetime dependence $P_{\mathrm{br}, i}(\tau)$ for each period $i$ of data taking was calculated, which takes into account the target thickness and experimental spectrum on $\pi^{+} K^{-}$or $\pi^{-} K^{+}$laboratory momenta. The maximum likelihood method has been applied

$$
L(\tau)=\exp \left(-U^{T} G^{-1} U / 2\right)
$$


MESON 2014 $-13^{\text {th }}$ International Workshop on Production, Properties and Interaction of Mesons

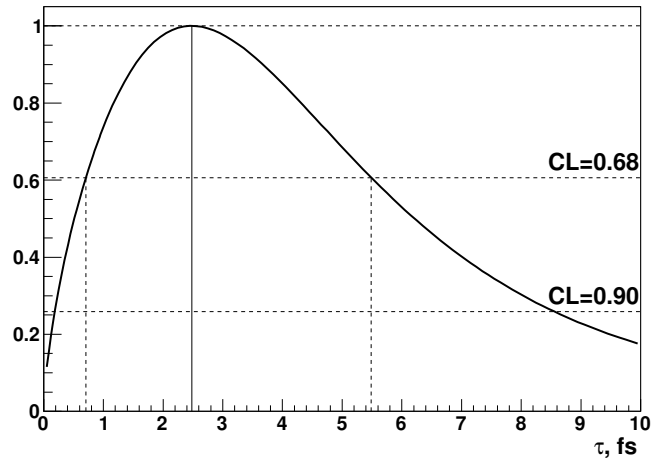

Figure 4. Likelihood function for $A_{\pi K}$ lifetime estimation based on both charged combinations $\pi^{+} K^{-}$and $\pi^{-} K^{+}$and all data periods combined, systematic uncertainties are included.

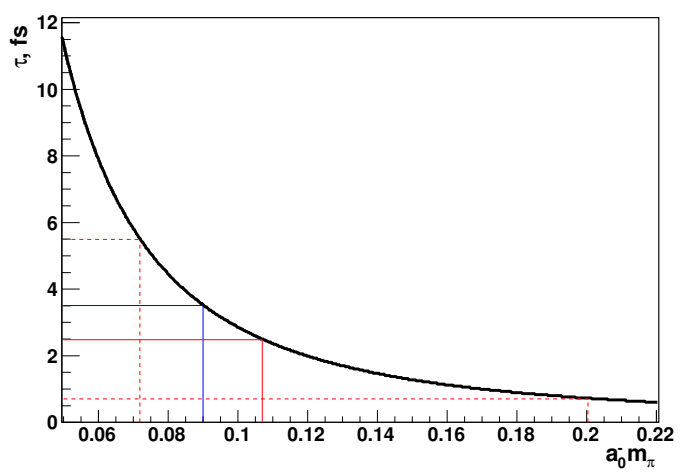

Figure 5. Dependence of $A_{\pi K}$ lifetime in the ground state $\tau$ on $\pi K$ scattering length $a_{0}^{-}$. Experimental result (red) is compared to theoretical prediction (blue).

where $U_{i}=\Pi_{i}-P_{\mathrm{br}, i}(\tau)$ is a vector of differences between measured $\Pi_{i}\left(P_{\mathrm{br}}\right.$ in Table 1$)$ and theoretical breakup probability $P_{\mathrm{br}, i}(\tau)$ for data sample $i$. The error matrix $G$ includes both statistical and systematic uncertainties [16]. The combined likelihood function $L(\tau)$ for both charge combinations is shown on Fig. 4. Its maximum yields the following estimation of $\pi K$ atom lifetime in the ground state:

$$
\tau=\left(\left.2.5_{-1.8}^{+3.0}\right|_{\text {stat }}-\left.0.1\right|_{\text {syst }}\right) \text { fs }=\left(\left.2.5_{-1.8}^{+3.0}\right|_{\text {tot }}\right) \text { fs. }
$$

The estimated ground state lifetime (7) corresponds to the $\pi K$ scattering length (1)

$$
\left|a_{0}^{-}\right| M_{\pi}=\frac{1}{3}\left|a_{1 / 2}-a_{3 / 2}\right| M_{\pi}=0.11_{-0.04}^{+0.09},
$$

to be compared with the theoretical predictions (Eq. 2, Fig. 5).

\section{Conclusions}

The analysis of $\pi K$ data collected by DIRAC experiment in 2008-2010 leads to the observation of $n_{A}\left(\pi^{-} K^{+}+\pi^{+} K^{-}\right)=178 \pm 49$ (3.6 sigma) characteristic atomic pairs of both charge combinations. The probability of $A_{\pi K}$ ionization (break-up) has been measured. Combined with the known dependence of break-up probability on atom lifetime, this results in a first measurement of the $\pi K$ atom lifetime in the ground state $\tau=\left(\left.2.5_{-1.8}^{+3.0}\right|_{\text {tot }}\right)$ fs. As the atom lifetime is related to a scattering length, a measurement of the S-wave isospin-odd $\pi K$ scattering length $\left|a_{0}^{-}\right|=\left(0.11_{-0.04}^{+0.09}\right) M_{\pi}^{-1}$ has been presented.

\section{Acknowledgements}

This work has been partially supported by Russian Foundation for Basic Research, research project 13-01-00060.

\section{References}

[1] B. Adeva et al. (DIRAC Collaboration), Phys. Lett. B 735 (2014) 288; arXiv:1403.0845. 
[2] J. Gasser, H. Leutwyler, Nucl. Phys. B 250 (1985) 465.

[3] G. Colangelo, J. Gasser, H. Leutwyler, Nucl. Phys. B 603 (2001) 125.

[4] J. Bijnens, P. Dhonte, P. Talavera, JHEP05 (2004) 036.

[5] B. Adeva et al. (DIRAC Collaboration), Phys. Lett. B 704 (2011) 24.

[6] J.R. Batley et al. (NA48/2 Collaboration), Eur. Phys. J. C 64 (2009) 589.

[7] J.R. Batley et al. (NA48/2 Collaboration), Eur. Phys. J. C 70 (2010) 635.

[8] S.M. Bilen'kii et al., Sov. J. Nucl. Phys. 10 (1969) 469.

[9] J. Schweizer, Phys. Lett. B 587 (2004) 33.

[10] P. Buettiker, S. Descotes-Genon, B. Moussallam, Eur. Phys. J. C 33 (2004) 409.

[11] L.L. Nemenov, Sov. J. Nucl. Phys. 41 (1985) 629.

[12] A. Sakharov, Zh. Eksp. Teor. Fiz. 18 (1948) 631; reprinted in Sov. Phys. Usp. 34 (1991) 375.

[13] C. Santamarina et al., J. Phys. B 36 (2003) 4273.

[14] M. Zhabitsky, Phys. At. Nucl. 71 (2008) 1040.

[15] B. Adeva et al. (DIRAC Collaboration), Phys. Lett. B 674 (2009) 11.

[16] V. Yazkov, M. Zhabitsky, DIRAC NOTE-2013-06 (cds.cern.ch/record/1628544). 